\title{
Lift Force Improvements for the Micromechanical Flying Insect*
}

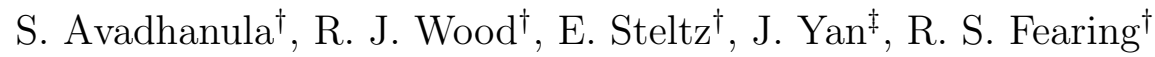 \\ $\dagger\{$ srinath, rjwood, ees132, ronf\}@eecs.berkeley.edu \\ $\ddagger$ josephy@ece.ubc.ca \\ ${ }^{\dagger}$ Department of EECS, University of California, Berkeley, CA 94720 \\ ${ }^{\ddagger}$ Department of ECE, University of British Columbia, Vancouver, Canada V6T 1Z4
}

\begin{abstract}
This paper presents some recent improvements in the fabrication and control of the Micromechanical Flying Insect (MFI), a centimeter sized aerial vehicle currently being developed at the University of California, Berkeley. We report a lift of $506 \mu N$ from a single wing, which is sufficient for a $100 \mathrm{mg}$ machine to lift itself off the ground. This lift matches very well with predictions based on quasi steady state models. We present some recent improvements in thorax fabrication leading to the development of a light weight platform $(\approx 100 \mathrm{mg})$, which generates $400 \mu N$ of lift with a single wing. We also present a new sensor mechanism which makes it possible to sense the motion of the actuators without having to add anything to the structure itself.
\end{abstract}

\section{Introduction}

Micro Aerial Vehicles (MAV) are an attractive platform for sensor deployment, search and rescue, reconnaissance etc. because of their very small size and high maneuverability. Early work on microrobotic flight was done by Shimoyama et al [1], while various aspects and approaches to micro aerial flight are being pursued by various groups $[3,6]$. The Micromechanical Flying Insect (MFI) project at UC Berkeley aims at building one such MAV with a wing span of about $25 \mathrm{~mm}$ and weighing about $100 \mathrm{mg}$. Fundamental work by Dickinson et al $[2,7]$ showed that insects depend on a complex interaction of unsteady state aerodynamics to generate the necessary forces for lift and maneuverability. In order to generate these forces, they showed that insect wings must be capable of 2 degrees of freedom (DOF) called flapping and rotation. For sufficient forces, the wing must be able to go through about $120^{\circ}$ of flapping and about $90^{\circ}$ of rotation.

${ }^{*}$ This work was funded by ONR MURI N00014-98-1-0671 and DARPA.
Early work on the MFI proposed a flexible (or accordion shaped) wing to generate these 2 DOF [4]. Later, a rigid wing approach was adopted to generate better aerodynamic forces and to enable easier fabrication. A detailed dynamic model of the mechanism was derived and a framework for optimizing the various fabrication parameters based on this model was constructed [9]. At the time however, limitations in fabrication technology limited the performance of the structures.

This report summarizes the improvements in various performance parameters for the MFI. Section 2 describes the improvements to the structural dynamics from an improved wing differential design. Section 3 describes a flight force measurement experiment and compares the measurement to predictions by a quasi steady state model. Section 4 presents a technique for directly measuring the lift and thrust forces of the newest version of the MFI. Section 5 describes a new sensor mechanism which enables the external measurement of actuator (and wing) displacements and describes how this sensor can be used to estimate the wing damping for the MFI.

\section{Improvements in Thorax Dynamics}

The major mechanical component of the MFI is the thorax which consists of two fourbar mechanisms which amplify the motion from the piezo electric actuators and a spherical wing differential mechanism to convert these amplified motions into wing flapping and rotation. One of the major recent improvements in the thorax is a change in the basic structure of the wing differential. Previously, the wing differential consisted of a modification to the basic spherical four-bar mechanism using a spherical joint composed of three flexures as shown in Fig. 1(a). Because this mechanism uses a larger number of flexures, it is able to tolerate greater fabrication misalignments without jamming, but suffers from too much stiffness. Recently, improved fabrication techniques with much better precision have 
enabled the use of proper spherical four-bars consisting solely of single $D O F$ flexural joints (See [16] for a treatment of spherical fourbars and [5] for a treatment of flexures). Since we use a smaller number of flexures, serial stiffness is increased allowing for greater power transmitted into the wing. Fewer flexures to prestress also means vastly improved fabrication times.

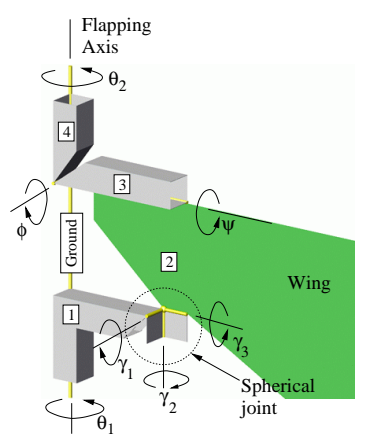

(a)

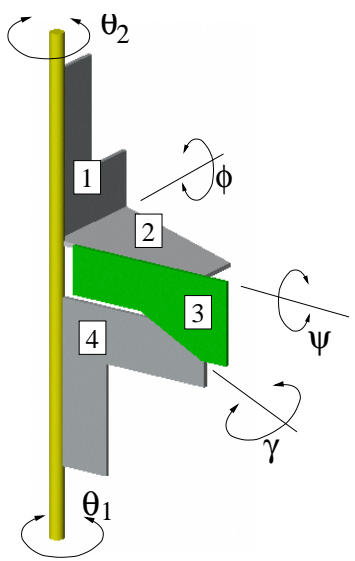

(b)
Figure 1: Improvement in Wing Differential Design (a) The wing differential mechanism design in [9] (b) The new wing differential mechanism design.

The dynamic behavior of the thorax however, remains the same as before because the change only manifests itself as a (favorable) lower differential stiffness without affecting anything else. This model was derived in $[8,9]$ and consists of a coupled $2 \times 2$ matrix which links the fourbar actuations (torques) to wing motion.

$$
\left[\begin{array}{l}
\theta_{1} \\
\theta_{2}
\end{array}\right]=\underbrace{\left[\begin{array}{ll}
G_{11}(s) & G_{12}(s) \\
G_{21}(s) & G_{22}(s)
\end{array}\right]}_{=: G(s)}\left[\begin{array}{l}
\tau_{1} \\
\tau_{2}
\end{array}\right]
$$

The diagonal terms of this transfer function are the drive transfer functions, while the off diagonal terms represent coupling between the fourbars through the wing differential. Ideally, we like the two drive transfer functions to be identical and the coupling to be negligible compared to the drive.

Advances in the fabrication of the fourbar and differential reported by Wood et al [10], have enabled these criteria to be met with much greater success than previous generations of the MFI which used a $\mu$-origami approach proposed in [14]. As a comparison, consider the behavior of the structure reported in [9] shown in Fig. 2(a), which shows a very coupled system arising from a large differential stiffness and a high inertia. The present generation of the MFI, whose performance is shown in Fig. 2(b), shows a remarkably low coupling, a high resonance and excellent DC motion. Although these are predictions, they have been experimentally verified on various occasions. See [9] for one of the experiments which verified these predictions.
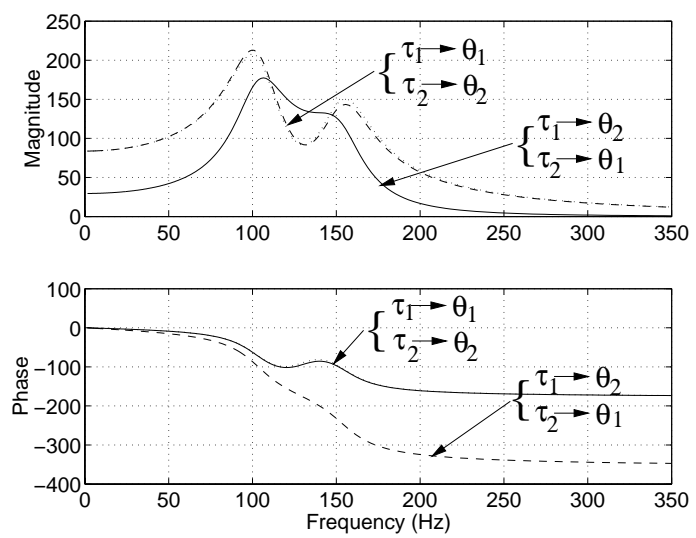

(a)
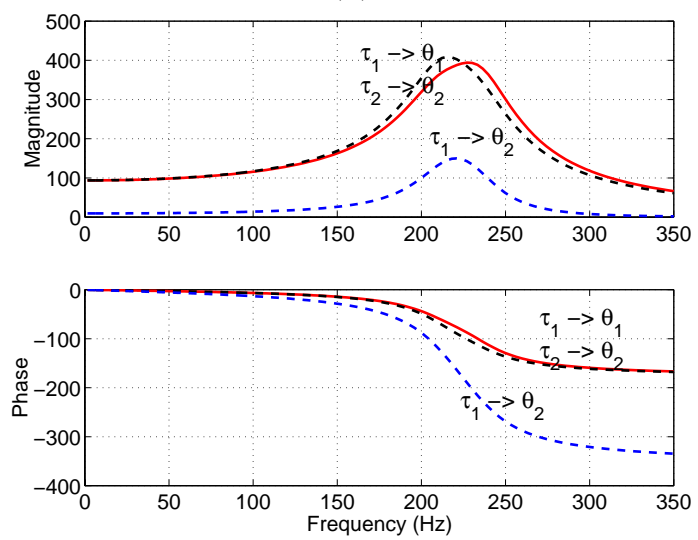

(b)

Figure 2: Predicted Frequency responses. (The magnitudes shown are the peak-peak wing displacements at 150V) (a) Thorax constructed in May 2002 (b) Thorax constructed in March 2003.

Table 1 shows the evolution of the inertial components of the MFI thorax over the past 2 years which has a large part to play in the improved dynamics. Table 2 show the evolution of the predicted wing power for the MFI based on its performance.

\section{Flight Force Measurement}

\subsection{Quasi Steady State Analysis}

Schenato et al. [12] and Sane and Dickinson [7] have derived a framework for calculating the expected wing 


\begin{tabular}{|l|l|l|l|}
\hline & $\begin{array}{l}12 \mu m \text { SS } \\
(\text { Aug 01) }\end{array}$ & $\begin{array}{l}6 \mu m \text { SS } \\
(\text { Jan 02) }\end{array}$ & $\begin{array}{l}40 \mu m \text { CF } \\
\text { (Sep 02) }\end{array}$ \\
\hline \hline Actuators & 3 & 3 & 3 \\
\hline Fourbar & 30 & 15 & 10 \\
\hline Differential & 10 & 8 & 8 \\
\hline Wing & 21 & 17 & 8 \\
\hline Total & 60 & 43 & 29 \\
\hline
\end{tabular}

Table 1: Evolution of thorax inertia parameters $\left(\mathrm{mg}-\mathrm{mm}^{2}\right)$ (The first row describes the major structural material at various stages of the project which changed from stainless steel (SS) to carbon fiber (CF).)

\begin{tabular}{|c|c|c|}
\hline & Wing Resonance & Wing Power \\
\hline \hline Aug 2001 & $100 \mathrm{~Hz}$ & $1.5 \mathrm{~mW}$ \\
\hline Jan 2002 & $120 \mathrm{~Hz}$ & $2.3 \mathrm{~mW}$ \\
\hline Sep 2002 & $160 \mathrm{~Hz}$ & $3 \mathrm{~mW}$ \\
\hline
\end{tabular}

Table 2: Evolution of MFI wing beat frequency and power

forces using a quasi-steady state model which assumes that the force equations derived for 2D thin aerofoils also hold for time varying 3D flapping wings. Yan and Fearing [13] have adapted this model to the MFI wings accounting for the specific wing morphology of the MFI and the fact that the MFI kinematics is not identical to insect wing kinematics as far as rotational axes go. This method is briefly outlined here. For the wing shown in Fig. 3(a), the instantaneous differential force contributions from a vertical wing blade element of thickness $\mathrm{d} r$ at a distance $r$ from the wing pivot are given as follows:

$$
\begin{aligned}
\mathrm{d} F_{\mathrm{tr}, \mathrm{N}}(t, r) & =\frac{C_{N}\left(\phi_{r}(t)\right) \rho c(r) U^{2}(t, r) \mathrm{d} r}{2} \\
\mathrm{~d} F_{\mathrm{tr}, \mathrm{T}}(t, r) & =\frac{C_{T}\left(\phi_{r}(t)\right) \rho c(r) U^{2}(t, r) \mathrm{d} r}{2} \\
\mathrm{~d} F_{\text {rot, } \mathrm{N}}(t, r) & =C_{r o t} \rho c^{2}(r) \dot{\phi}_{r} U(t, r) \mathrm{d} r
\end{aligned}
$$

where the "tr" and "rot" subscripts indicate whether the contribution is due to translational or rotational motion, the "N" and " $\mathrm{T}$ " subscripts indicate that the direction is either normal or tangential to the wing (notice that the rotational component is always assumed as a normal pressure force), $\rho$ is the density of air, $\phi_{r}$ and $\phi_{f}$ are the wing rotation and flapping angles, $U$ is the velocity of the blade element, $c$ is the wing chord width, $C_{N}$ and $C_{T}$ are dimensionless translational force coefficients for the normal and tangential components to the wing (both are functions of $\phi_{r}$ ), and $C_{r o t}$ is the rotational force coefficient. $C_{r o t}$ is taken to be a function of the non-dimensional axis of rotation $\hat{x_{0}}$ which varies linearly from zero at the leading edge to unity at the trailing edge:

$$
C_{\text {rot }}=\pi\left(\frac{3}{4}-\hat{x_{0}}(r)\right)
$$

It is important to note here that $\hat{x_{0}}$ is usually taken to be a constant but this does not seem to adequately consider how the position of the blade element relative to the rotation axis changes the force so it has been written here explicitly as a function of $r$.

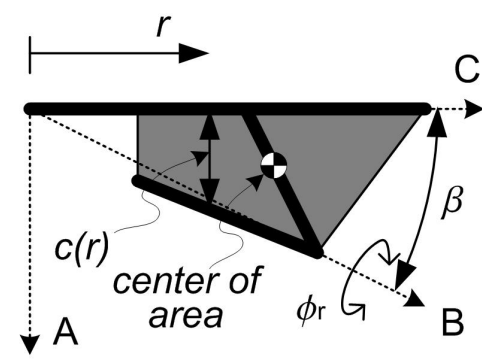

(a) Geometry of MFI wing

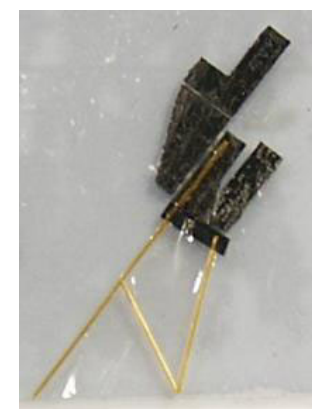

(b) Carbon Fiber Differential and Wing
Figure 3: Wing shape.

In our setup, the body is stationary so this simplifies the force estimate as there are no body dynamics to consider. Setting $U(t, r)=\dot{\phi}_{f}(t) r$, equations (2)-(4) can be integrated:

$$
\begin{aligned}
F_{t r, N}(t) & =\frac{\rho C_{N}\left(\phi_{r}(t)\right) \dot{\phi}_{f}^{2}(t)}{2} I \\
F_{t r, T}(t) & =\frac{\rho C_{T}\left(\phi_{r}(t)\right) \dot{\phi}_{f}^{2}(t)}{2} I \\
F_{r o t, N}(t) & =\rho \pi \dot{\phi}_{r}(t) \dot{\phi}_{f}(t) \tilde{I}
\end{aligned}
$$

where $I$ is the second moment of area of the wing about axis $\mathbf{A}$ and

$$
\tilde{I}=\int\left(\frac{3}{4}-\hat{x}_{0}(r)\right) c^{2}(r) r \mathrm{~d} r
$$

The wing of Fig. 3 has center of area located at $\left(x_{c a}, y_{c a}\right)=(6.70,-1.49) \mathrm{mm}$ and the chord length is parameterized according to the equation:

$$
c(r)= \begin{cases}\frac{2}{5} r+\frac{4}{5} \mathrm{~mm} & \text { if } 3 \mathrm{~mm} \leq r<8 \mathrm{~mm} \\ \frac{4}{3} r+14 \frac{2}{3} \mathrm{~mm} & \text { if } 8 \mathrm{~mm} \leq r \leq 11 \mathrm{~mm} \\ 0 & \text { otherwise }\end{cases}
$$

For this wing, $I=1020 \mathrm{~mm}^{4}$ and the value of $\tilde{I}$ can be calculated as $-140 \mathrm{~mm}^{4}$. 


\begin{tabular}{|c|c|}
\hline Stroke angle (pk-pk) & $125^{\circ}$ \\
\hline Rotation angle (peak) & $\pm 45^{\circ}$ \\
\hline Measured Lift force (mean) & $506 \mu N$ \\
\hline Predicted Lift force (mean) & $526 \mu N$ \\
\hline
\end{tabular}

Table 3: Results from flight force experiment on $03-\delta$

\subsection{Experimental Setup}

For this particular experiment, we used $03-\delta$, one of the latest versions of the MFI. This version utilizes oversized PZT-5H actuators each weighing about $120 \mathrm{mg}$ each. It should be noted however, that the final scale PZN-PT actuators (each of which weighs about $20 \mathrm{mg}$ ) provide the same performance as the PZT-5H. Since the entire structure is too heavy for being placed on a body force sensor as described in section 4, we utilize a commercially available precision weighing balance to measure the lift force. We use the AAA 250 from Adam Equipments with a repeatability of $2.5 \mu \mathrm{N}$ and a settling time of approximately $20 \mathrm{~s}$ in practice. The technique is similar to the one described in [13].

For this experiment, we used simple sine wave drives to the two actuators at $150 \mathrm{~Hz}$. The drive signals were adjusted till a flapping angle of $120^{\circ}$ and a rotation of $\pm 45^{\circ}$ were obtained. By taking a movie of the wing motion from the top view and identifying various distinct wing features, the wing trajectory was computed. Fig. 4 shows a sample of the frames from the movie and Fig. 5 shows the trajectory extracted from such image sequences. From this it is easy to identify some key features of the wing motion. Firstly, we see that the wing moves through about $120^{\circ}$ of flapping and that the rotation timing is well suited for generating lift. We see that the wing flip occurs before the end of the downstroke at one end and right near the end of the stroke for the upstroke. Moreover, the wing maintains a reasonable angle of attack for a good percentage of the downstroke which is also important from a lift perspective.

When this trajectory is used to predict the wing forces according to equations (6)-(8), we obtain a wing lift trajectory over the wing beat as shown in Fig. 6 . The predicted mean lift is found to be $526 \mu N$, which is in remarkable agreement with the measured lift force of about $506 \mu N$. Table 3 presents a summary of the results from this experiment.

\section{Direct MFI Force Measurements}

This section describes a flight force experiment performed on $03-\gamma$, a final scale version of the MFI

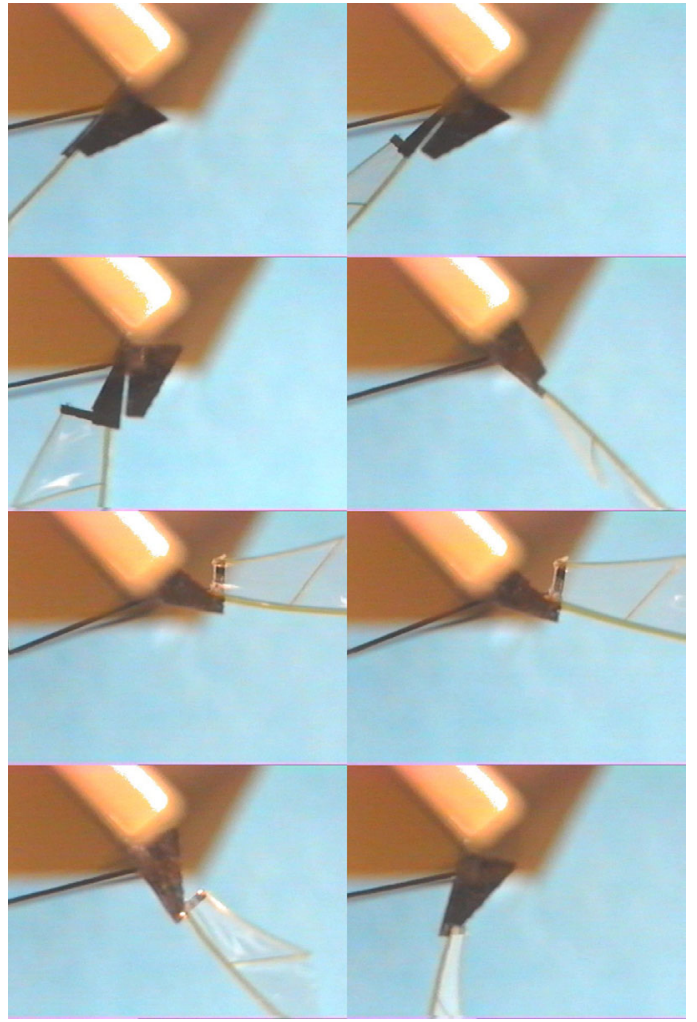

Figure 4: Motion of $03-\delta$ at $150 \mathrm{~Hz}$ captured by illuminating the wing with a strobe light. The frames are arranged in increasing order from left to right and top to bottom.

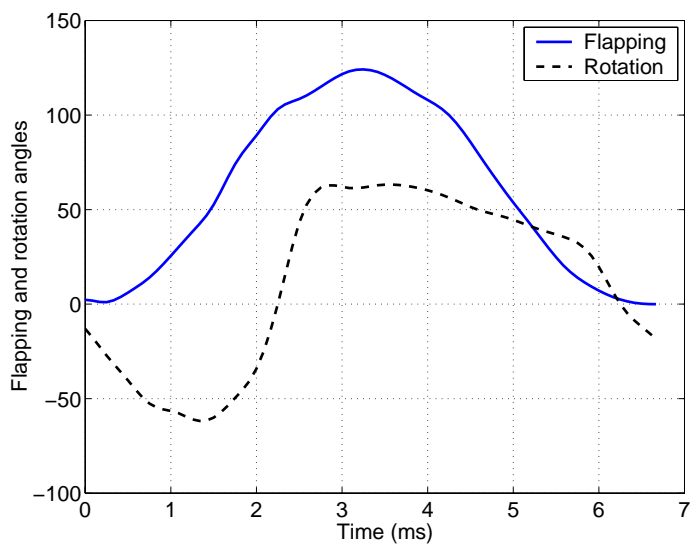

Figure 5: Wing Trajectory of $03-\delta$

fabricated using techniques described in [10] weighing approximately $80 \mathrm{mg}$. To measure the actual forces the MFI is generating on a wingbeat per wingbeat basis, it is placed upon a $2 D O F$ force platform. For this test, only one half of the MFI is necessary, thus the one wing, $2 D O F$ structure shown in figure 8 is used. 


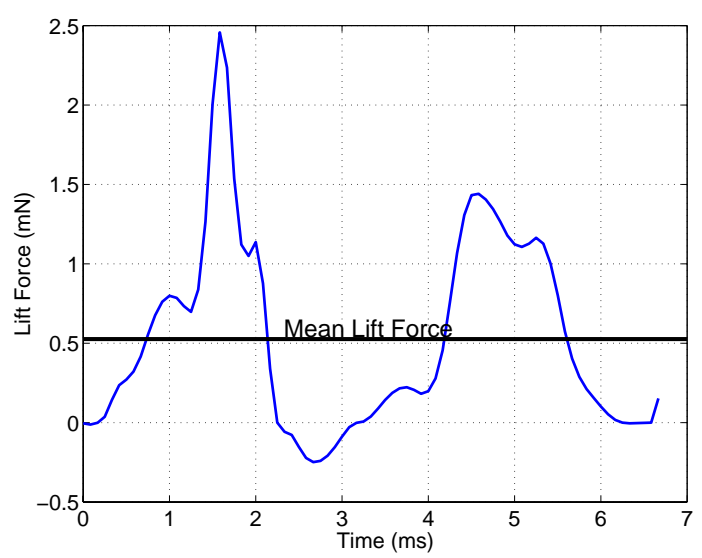

Figure 6: Predicted Lift Force

This platform allows the lift force and drag or thrust force to be measured independently and in real time [11]. This was done previously with real insects, however until recently the mass of the MFI has limited the use of this structure.

\subsection{Open Loop Control Strategy}

We employed an open loop feedforward control strategy to generate the drive voltages for this structure. This consists of using strain gage sensors mounted at the base of the actuator to identify the thorax transfer function. The details on how to accomplish this are presented in [9]. A pseudo-inverse of this plant is then calculated. The desired wing trajectory is then given as an input to the plant generating the required drive voltages as shown in Fig. 7 .

To test the lift capabilities of this structure, the wing is driven under kinematic control with a desired trajectory of $\pm 20^{\circ}$ phase difference (which gets converted into $\pm 45^{\circ}$ of wing rotation through the differential transmission) superimposed upon $120^{\circ}$ of flapping motion for the leading and lagging wing spars respectively. Due to structural limitations, we obtain a wing trajectory of $\pm 20^{\circ}$ of rotation superimposed on $70^{\circ}$ of flapping. This structure with these kinematics, driven at its resonant frequency of $170 \mathrm{~Hz}$ gives an average lift of approximately $400 \mu N$ and the resulting force traces are given in figure 9. Thus this one wing structure gives a lift just shy of half the total necessary force required for the MFI to hover. This test platform provides an important tool for future testing. For a given kinematic parameterization, the resulting lift and thrust forces can be obtained in real time. This will allow searches over these parameter spaces to be quicker, putting the structure through less fatigue and obtaining more reliable results.

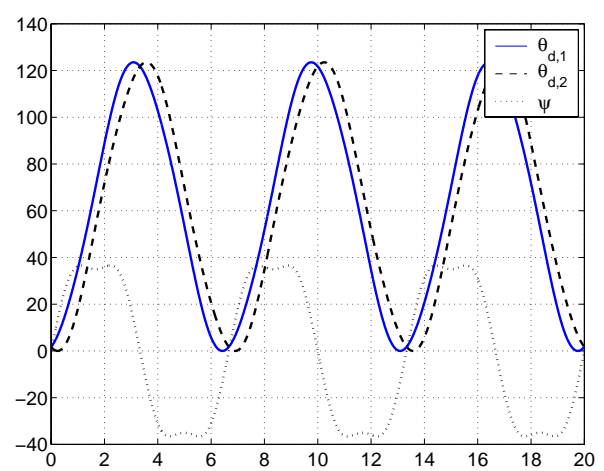

(a) Desired Wing Trajectory.

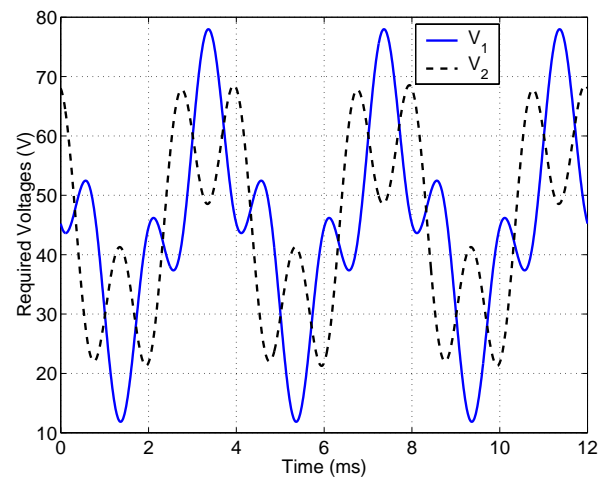

(b) Wing trajectory fed into

the pseudo inverse generates the required drive voltages to the actuators.

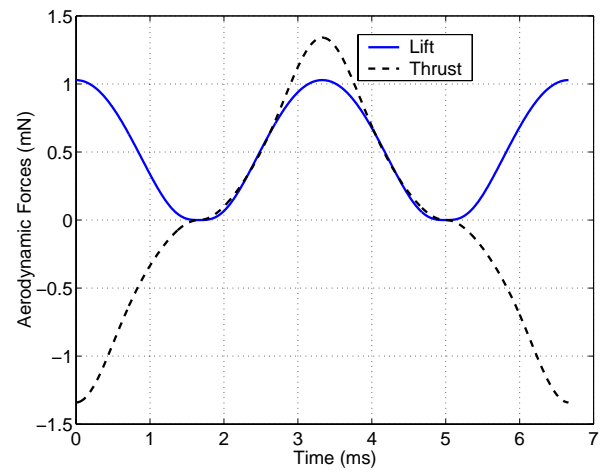

(c) Simulated wing forces showing a mean lift of about $510 \mu N$.

Figure 7: Feedforward Control of MFI thorax

\section{Optical Sensing}

Recent advancements in sensing and characterization of thorax structures have improved prototype fabrication speed and performance. Specifically, the adoption of optical sensing to observe actuator (and therefore wing) deflection allows external sensing without restrictions on the size and weight of the equipment. One mobile unit can also be used on many 


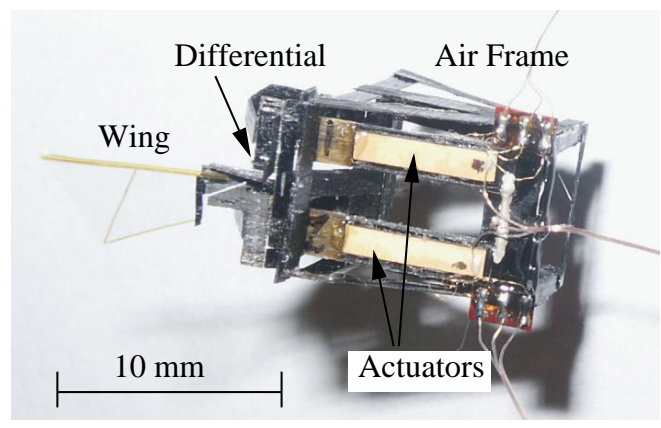

Figure 8: One wing, 2DOF MFI.

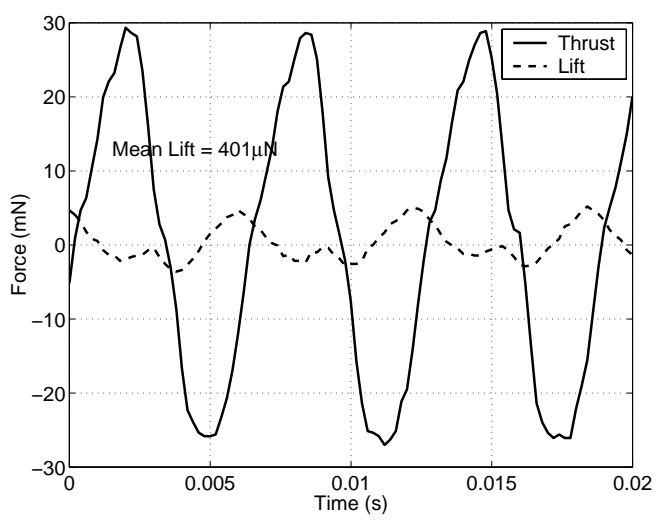

Figure 9: Measured lift and drag forces.

prototypes as opposed to the former method of strain gauge mounting, which needs to be done for each model.

The technique employed aims an infrared beam at the back of the actuators and receives the reflected signal using a photo-transistor. Careful adjustment can lead to linear voltage output of the sensor with respect to actuator deflection. After proper amplification, actuator deflections in the range of $1 \mu m$ can be successfully detected.

This new sensing technique was employed on a 1DOF fourbar/wing to both demonstrate the ease of characterization it can introduce and to verify its accuracy. The setup employed appears in Fig. 10. Using a dynamic signal analyzer, a bode plot of the input voltage to the actuator vs the output of the optical sensor was created. The plot was then converted into wing stroke by measuring the wing angle at $10 \mathrm{~Hz}$ at the same voltage signal as used during the frequency sweep. Because the sensor is linear, the magnitude of the bode plot can be calibrated by multiplying by the constant value between the signal analyzer output at $10 \mathrm{~Hz}$ and the wing stroke measured with a microscope camera at that frequency. The calibrated bode plot appears in Fig. 11.

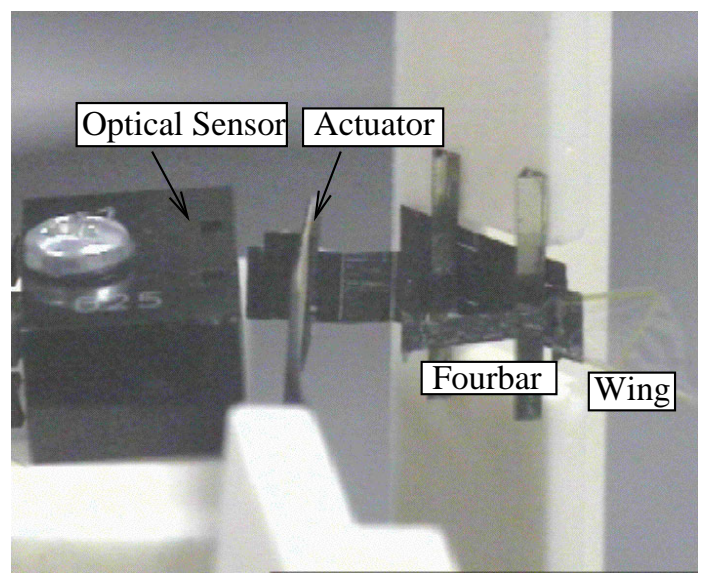

Figure 10: One DOF optical sensing setup
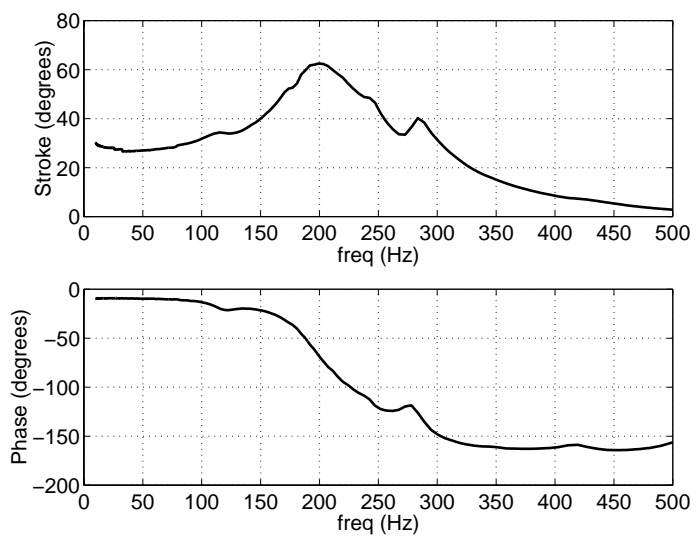

Figure 11: Bode plot for high amplitude flapping

To verify the accuracy of the procedure using optical sensing, the system's stiffness, transmission ratio, $\mathrm{Q}$, and wing damping were calculated and compared with expected values. As can be easily observed, the Bode plot in Fig. 11 was produced using a high amplitude input signal, so one expects a low Q for this system with an aggressively flapping (and therefore highly damped) wing. A Q of 2.21 can be directly observed from the resonant peak in the bode plot. Knowing the stiffness and transmission ratio (measured at DC) to be

$$
\begin{gathered}
k_{\text {total }}=280.5 \mathrm{~N} / \mathrm{m} \\
T=2.6 \times 10^{3} \mathrm{rad} / \mathrm{m}
\end{gathered}
$$

One can use the observed resonant frequency (204 $\mathrm{Hz}$ ) to find the inertia of the system 


$$
J=\frac{k_{\text {total }}}{w_{r}^{2}}=1.71 \times 10^{-4} \mathrm{~kg}
$$

and finally the wing damping can be found by

$$
b=\frac{1}{T^{2}} \frac{\sqrt{k_{\text {total }} J}}{Q}=14.6 \times 10^{-9} \mathrm{Nms} / \mathrm{rad}
$$

Preliminary estimates of the wing damping based on insect weight were reported in [4] as $8.65 \times$ $10^{-9} \mathrm{Nms} / \mathrm{rad}$. Since that estimate was for an insect wing beating at $150 \mathrm{~Hz}$, while this experiment had the wing beating at $204 \mathrm{~Hz}$, the higher value seems to be in good agreement.

\section{Conclusions}

This paper presented an important milestone in the MFI project. We report a lift of $500 \mu N$, the "breakeven" point for the first time. The wing differential mechanism has also crossed the elusive $120^{\circ}$ flapping and $90^{\circ}$ rotation for the first time. Flight force measurements validate several important things about the MFI. Firstly that generation of adequate lift is feasible with the present mechanism and actuator design. Next it also provides important corroboration to the quasi steady state models derived in [12]. New materials and fabrication techniques have made possible real time control and flight force sensing of the MFI thorax yielding a force of $400 \mu N$ from a single wing robot weighing about $80 \mathrm{mg}$.

\section{Acknowledgments}

The authors would like to acknowledge Luca Schenato for helpful discussions on insect wing aerodynamics, Manas Menon for valuable help in the fabrication of the MFI thorax and Domenico Campolo for helpful discussions on wing control.

\section{References}

[1] I. Shimoyama, H. Miura, K. Suzuki, and Y. Ezura, "Insect-like microrobots with external skeletons," IEEE Control Sys. Magazine, 13:37-41, February 1993.

[2] M.H. Dickinson, F-O. Lehmann, and S.P. Sane, "Wing rotation and the aerodynamic basis of insect flight," Science, 284:1954-1960, June 1999.

[3] A. Cox, E. Garcia, and M. Goldfarb, "Actuator development for a flapping microrobotic MAV," In SPIE Microrobotics Symp., pages 102-108, Boston, MA, Nov 1998.
[4] R.S. Fearing, K.H. Chiang, M.H. Dickinson, D.L. Pick, M. Sitti, and J. Yan, "Wing transmission for a micromechanical flying insect," IEEE International Conference. on Robotics and Automation, pages 15091516, San Francisco, CA, April 2000.

[5] M. Goldfarb, J.E. Speich, "A Well-behaved revolute flexure joint for compliant mechanism design," Transactions of the ASME, Vol 121, pages 424-429, September 1999 .

[6] T. Nick Pornsin-Sirirak, Y.C. Tai, H. Nassef and C.M. Ho, "Titanium-Alloy MEMS Wing Technology for A Micro Aerial Vehicle," Application, Sensors and Actuators, A: Physical, vol. 89, no. 1-2, pp. 95-103, Mar. $20,2001$.

[7] S.P. Sane and M.H. Dickinson. "The aerodynamic effects of wing rotation and a revised quasi-steady model of flapping flight", Journal of Experimental Biology, 1087-96, Vol 205, 2002

[8] S. Avadhanula. "The Design and Fabrication of a dynamically tuned MFI thorax", MS Thesis, University of California, Berkeley, Dec 2001.

[9] S. Avadhanula, R.J. Wood, D. Campolo and R.S. Fearing. "Dynamically tuned design of the MFI thorax", Proceedings of the IEEE International Conference on Robotics and Automation, Washington, DC, May 2002.

[10] R.J. Wood, S. Avadhanula and R.S. Fearing. "Microrobotics using Composite Materials", To appear: IEEE International Conference on Robotics and Automation, Taipei, Taiwan, May 2003.

[11] R.J. Wood and R.S. Fearing. "Flight force measurements for a micromechanical flying insect", IEEE Conference on Intelligent Robots and Systems, Maui, HI, Oct. 29-Nov. 3, 2001.

[12] L. Schenato and X. Deng and S. Sastry. "Virtual Insect Flight Simulator (VIFS): A Software Testbed for Insect Flight", Proceedings of IEEE International Conference on Robotics and Automation, Seoul, Korea, May 21-30, 2001.

[13] J. Yan and R.S. Fearing, "Force Map Characterization for a Micromechanical Flying Insect", submitted to: IEEE International Conference on Intelligent Robots and Systems, Las Vegas, NV, October 27-21, 2003.

[14] E. Shimada, J.A. Thompson, J. Yan, R.J. Wood, and R.S. Fearing, "Prototyping millirobots using dextrous microassembly and folding," Symposium on Microrobotics ASME International Mechanical Engineering Cong. and Expo., Orlando, FL, Nov 2000.

[15] J. Yan, S. Avadhanula, J. Birch, M.H. Dickinson, M. Sitti, T. Su and R.S. Fearing, "Wing Transmission for a micromechanical flying insect," Journal of $\mathrm{Mi}$ cromechatronics, Vol. 1, No. 3, pp. 221-237, 2002.

[16] D. A. Ruth and J. M. McCarthy, "The Design of Spherical 4R Linkages for Four Specified Orientations," Computational Methods in Mechanisms, ed. J. Angeles, Spring-Verlag, 1998. 